\title{
HAES-Infusionen sind wirksam beim Hörsturz : Ergebnisse einer placebokontrollierten Doppelblindstudie an 210 Patienten
}

\author{
Ralph Mösges*, Eckart Klemm**, Frank Bepperling***, Martin Burschka**** \\ *Institut für medizinische Statistik, Informatik und Epidemiologie, Universitätsklinikum Köln, 50924 Köln \\ **HNO-Klinik Krankenhaus Dresden-Friedrichstadt, Friedrichstr. 41, 01067 Dresden \\ ***Fresenius Kabi Deutschland GmbH, Else Kröner Str. 1, 61352 Bad Homburg v.d.H.
}

Einleitung

Die Leitlinien der Fachgesellschaft zum Hörsturz sehen in der Hämodilutionstherapie eines der allgemeinen Therapieprinzipien. Als Präparat der Wahl wird die Hydroxyethyl-Stärke (HES) eingesetzt. Die hier untersuchte Tetrastärke HES 130/0.4 zeichnet sich durch ein geringeres Molekulargewicht von 130000 Dalton und einen geringeren Substitutionsgrad $(0,4)$ als ältere Vorgänger aus, mit dem Ziel erhöhter renaler Exkretion und geringerer Gewebseinlagerung. Dadurch wird das Auftreten des substanzspezifischen Pruritus reduziert.

\section{Methodik}

Patienten mit unilateralem Hörsturz von mindestens 20 und höchstens $95 \mathrm{~dB}$ in mindestens zwei Frequenzen des Hauptsprachbereiches wurden in diese placebokontrollierte doppelblinde Dosisfindungsstudie eingeschlossen und sechs Tage lang mit Infusionen von $750 \mathrm{ml}$ behandelt, die entweder $45 \mathrm{~g}, 30 \mathrm{~g}$ oder $15 \mathrm{~g}$ HES 130/0.4 oder aber fünfprozentige Glucoselösung enthielten. Diese war als Placeboersatz gewählt worden, da sie sich visuell von der HES-Lösung nicht unterschied. Vor Beginn der Behandlung, nach drei, sieben und 90 Tagen wurden Reintonaudiogramme angefertigt.

\section{Ergebnisse}

210 Hörsturzpatienten wurden in 25 Zentren im Zeitraum zwischen November 2000 und Dezember 2002 in der Studie behandelt. Bei 208 auswertbaren Patienten fanden sich für das Hauptzielkriterium „Hörgewinn an Tag 7“ zwar numerische Vorteile für die HES-Gruppen gegenüber Placebo, jedoch keine statistisch signifikante Überlegenheit. Auffällig waren aber Unterschiede in der langfristigen Hörerholung zwischen Patienten, die innerhalb von $48 \mathrm{~h}$ nach dem Hörsturz zur Behandlung kamen (4,7\% ohne Verbesserung innerhalb von 90 Tagen) und jenen, die erst später behandelt wurden $(21,5 \%$ nach 90 Tagen ohne Besserung). Auch Patienten mit einem systolischen Blutdruck $>140 \mathrm{mmHg}$ hatten geringere Chancen einer Erholung. Für jene Patienten, die diesen Risikofaktor aufwiesen und sich später als $48 \mathrm{~h}$ nach Symptombeginn in die Behandlung begeben hatten, waren in der Placebogruppe nach einer Woche $46 \%$ noch ohne Hörbesserung, während in der 4\%-HES-Gruppe nur $13 \%$ ohne Besserung blieben ( $p=0,025$ für eine zumindest partielle Hörerholung unter HES am Tag 7). Die neuartige HES-Lösung erwies sich insgesamt als nebenwirkungsarm, insbesondere trat der substanztypische Pruritus nur temporär bei 4 von 157 mit HES 130/0.4 Behandelten auf.

\section{Schlussfolgerung}

Hörsturzpatienten ohne erhöhten systolischen Blutdruck und solche die sich innerhalb von zwei Tagen nach dem Ereignis behandeln lassen, haben eine nahezu 100 \%ige Chance einer Hörverbesserung unter i.v. Flüssigkeitssubstitution. Bei Patienten, bei denen der Hörsturz am dritten Tag noch persistiert und jenen, die einen erhöhten Blutdruck aufweisen, führt die i.v. Gabe von $750 \mathrm{ml} 4$ \%iger HES 130/0.4 einmal täglich über sechs Tage zu einer wesentlichen Verbesserung der langfristigen Prognose des Hörvermögens.

Wir danken den Mitgliedern der Studiengruppe

In Deutschland: D. Adler / U. Fröschl, Berlin; K. Frese / J. Schmelzer, Kiel; E. Gehrking / T. Berg, Lübeck; T. Günzel / C. Erle, Potsdam; H. Gudziol / K. Sahm, Jena; W. Heppt / J. Arndt, Karlsruhe; K. Hörmann / N. Hammerschmitt, Mannheim; O. Kaschke, Berlin; B. Klee, Brandenburg; S. Petzold Dresden-Friedrichstadt; C. Reißner / K. Sedighi, Heidelberg; R. Weber/S. Rudolph, Stuttgart; J.A. Werner / A. Teymoortash, Marburg;

In der Tschechischen Republik: J. Betka, Praha; J. Klačanský, Olomouc; R. Kostřica, Brno; A. Pellant, Pardubice; J. Vokurka, Hradec Kralove;

In Rumänien: S. Baschir, Arad; M. Bera, Alba Iulia; G. Comşa, Constanta; M. Cosgarea, Cluj Napoca;

In Österreich: F. Heger, Linz; P. Löffler, Linz; J. Meindl, Linz.
Objective: Obtain first data on HES 130/0.4 (hydroxyethyl starch) as monotherapy in patients with acute idiopathic sudden sensorineural hearing loss (ISSNHL). Design: Randomised, double-blind, phase-II, dose-finding study. Setting: 25 ENT centres in Germany, the Czech Republic, Romania, and Austria. Patients: 208 inpatients with first-time ISSNHL of ? $20 \mathrm{~dB}$ at ? 2 frequencies and ? 95 $\mathrm{dB}$ at all of the speech frequencies $(0.5,1.0,2.0,3.0,4.0 \mathrm{kHz}$ ) with respect to the other (normal) ear for up to $7 \mathrm{~d}$. Interventions: Infusion of $750 \mathrm{~mL} / \mathrm{d}$ with HES $45 \mathrm{~g} / \mathrm{d}$ (group H), $30 \mathrm{~g} / \mathrm{d}(\mathrm{M}), 15 \mathrm{~g} / \mathrm{d}(\mathrm{L})$, auditory threshold from baseline to Day 7 . Results (medians): Average hearing loss at baseline was $24 \mathrm{~dB}$ and infusions started $2 \mathrm{~d}$ after ISSNHL-onset. No relevant group difference was observed in hearing gain or side effects - including pruritus. Half of all patients recovered completely by Day 7 . Post-hoc analysis: In patients who had a treatment lag $<48 \mathrm{~h}$ and blood pressure RRsyst $<140$ $\mathrm{mmHg}$, hearing at Day 90 had improved in all 28 cases under placebo, whereas in patients, with treatment lag $>48 \mathrm{~h}$ and/or blood pressure RRsyst? $140 \mathrm{mmHg}$ the risk of failing to improve under placebo was $(7 / 24,29.2 \%)$. Comparing all 118/208 (51.9\%) patients at such risk, outcome at Day 7 was markedly better in all HES-groups than in group $G$ (Kruskal-Wallis $p=0.0221$ ). Conclusion: In the prospective analysis, all treatment groups were equivalent including side effects. In the post-hoc analysis, ISSNHL-patients at risk of not improving under placebo i.e. patients starting treatment later
than $48 \mathrm{~h}$ after IISNHL-onset and/or with elevated blood pressure, recovered markedly better under HES-infusions.

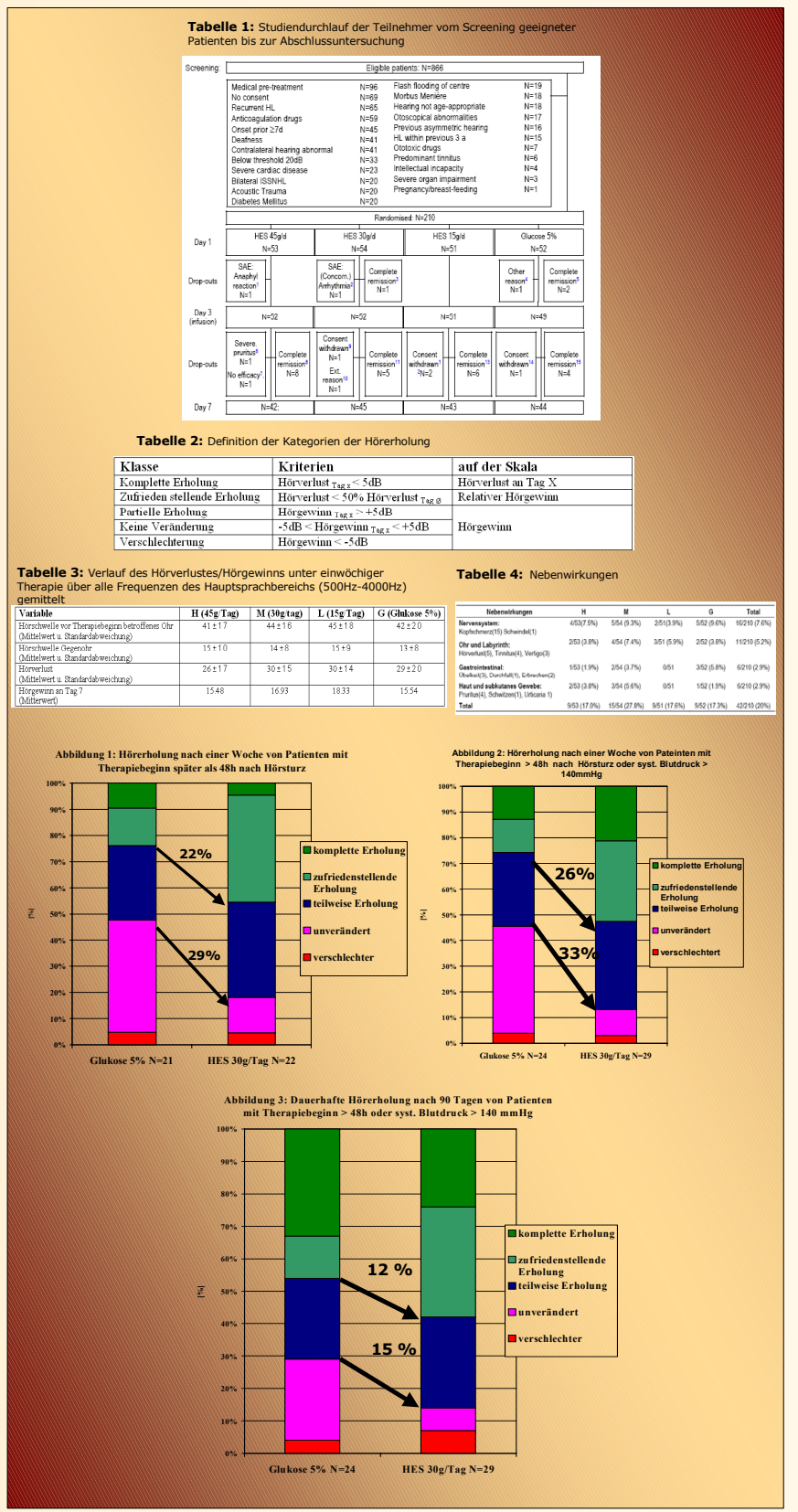

